\title{
Management of Incidental Adenoma Malignum of the Cervix: A Case Report
}

\section{Federico Ferrari ${ }^{1 *}$, Martina Ratti ${ }^{2}$, Valentina Zizioli ${ }^{1}$ and Franco Odicino ${ }^{2}$}

${ }^{1}$ Department of Obstetrics and Gynecology of Brescia, Civil Hospital of Brescia, Italy

${ }^{2}$ Department of Obstetrics and Gynecology, University of Brescia, Italy

${ }^{*}$ Corresponding author: Federico Ferrari, Department of Obstetrics and Gynaecology of Brescia, Civil Hospital of Brescia, 25123, Italy, Tel: + 39-3402729765; Fax: 390303996018; E-mail: f.ferrari.obgyn@gmail.com

Received date: 07 November, 2016; Accepted date: 15 December, 2016; Published date: 16 December, 2016

Citation: Ferrari F, Ratti M, Zizioli V, et al. Management of Incidental Adenoma Malignum of the Cervix: A Case Report. Gynecol Obstet Case Rep. 2016, 2:3.

\section{Abstract}

Background: Minimal deviation adenocarcinoma (MDA, also reported as Adenoma Malignum) of the cervix is a rare variant of cervical carcinoma. It is thought to represent about $1 \%$ to $3 \%$ of all cervical adenocarcinomas. The symptoms can be multifarious and aspecific, however the most peculiar and recurrent is a persistent watery-mucoid vaginal discharge. Because of the symptoms, patients are often referred to a general gynecologist and invariably further examinations are carried out, but not always a precise diagnosis is obtained. Thus, the patients are usually candidated for a surgical non-conservative treatment, mainly to relief the symptoms. Albeit its rareity this cancer is highly aggressive, hence the need for oncologically appropriate approach. Since extremely low incidence of this cancer the majority of general gynecologist will fail in recognise "hints symptoms" and therefore cannot offer a correct treatment.

Case presentation: We present a case of a caucasian patient, aged 45 years old, multipara and with profused watery vaginal discharge since half an year. According to anamnestic fitting, gynecological examinations were negative and a recent pap smear was reported as negative. Ultrasound scans described few cysts compatible with Nabothian cysts within an otherwise normal uterus and cervix. MRI of the pelvis confirmed the presence of the cystic morphology of the cervix. In view of the persistent symptomatology without a raised suspect of malignancy, the patient underwent an extrafascial hysterectomy and consensual bilateral salpingooophorectomy because of her age. Histopathology reported differential diagnosis among lobular endocervical glandular hyperplasia versus adenoma Malignum of the cervix. Patient has been therefore referred to the Gynecological Oncology service at our hospital. Histopathology review confirmed MDA of the cervix with a maximum size of $23 \mathrm{~mm}$, negative margins and without lymph-vascular invasion. Multidisciplinary Tumor Board suggested two equivalent options for appropriate treatment: adjuvant external beam radiotherapy of the pelvis with vaginal brachytherapy versus robotic bilateral parametrectomy and lymphnodal pelvic dissection for a proper staging. Further surgical approach has been performed and histopathology confirmed a stage FIGO Ib1. Patient has been therefore sent for routine follow-up and after 10 months she is cancer free.

Conclusion: Referring to tertiary hospitals a rare malignancy as MDA for primary treatment is the best practise. However even in case of incidental finding or misdiagnosis, an early and timely referring can hopefully reduce errors and morbidity in the primary treatment.

Keywords: Adenoma Malignum; Cervical cancer; Cervix; Minimal deviation carcinoma

\section{Background}

According to WHO classification since 2003 [1] Adenoma Malignum (AM) of the cervix is actually known as minimal deviation adenocarcinoma (MDA). Estimated incidence is $1 \%$ to $3 \%$ of all cervical adenocarcinoma and apparently without any age preferences [2]. Symptoms of this cancer are quite typical when present [3]. The most common symptom is a mucoid-watery vaginal discharge, often associated with a recurrent and irregular vaginal bleeding due or not to contact of the cervix. Pelvic pain and bloating and swelling abdomen can be present. In literature, there are reports of ancillary symptoms as severe electrolyte disturbances (profound iponatriemia) as consequence of the continuous watery discharge [4] or confundent symptoms as a presumed onset of urinary incontinence [5]. This type of cancer is frequently linked with Peutz-Jeghers syndrome (PJS) [6], whilst there isn't any demonstrated connection with the presence of HPV.

Since its rarity and peculiarities there aren't effective screening test, hence the challenge of a pre-operative diagnosis. Most of the time neither a punch or a cone biopsy of the cervix can reveal this malignancy and also colposcopic examinations might fail to detect any linked abnormalities of the cervix. Majority of the women affected by MDA have a pap smear showing typical glandular cells. In fact these lesions 
have mucin-rich cysts and are usually situated deep in the cervix and therefore exfoliated glandular cells are not uncommon [7]. Pathological diagnosis of MDA is a challenge [8] even for a skilled pathologist. It's a well differentiated adenocarcinoma with an endophytic growth and with a complex immunochemistry recognition pattern [9]. Most of the time clinical diagnosis of MDA is extremely difficult since the presence of cervical cysts is common. In fact Nabothian cysts are frequent and silent retention cysts of the uterine cervix with no particular intervention required [10]. Despite the non-aggressive histopathology pattern and the usual welldifferentiated cytology, these tumors have been described as highly malignant with a rapid lethal outcome (Hence the suggestive name "Adenoma Malignum"). A proper oncological treatment has to be delivered, especially in case of incidental diagnosis.

\section{Case Presentation}

In this case report [11] we present a caucasic patient of 45 years old in premenopausal status, who previously delivered two babies (multipara), complaining about a persistent watery vaginal discharge since half an year ( 6 months). Management of this patients has been carried out by a general gynecologist and therefore anamnestic fittings and exams reports have been collected. Multiple physical and gynecological examinations revealed normal sterna genitalia and an enlarged cervix reported as "grossly normal" for a multipara. A manifest collection of fluid in the vagina cannot be highlighted, not either an active cervical discharge or bleeding. In fact only a para-physiological vaginal fluid has been described during specular examination. A negative pap smear performed one year before onset of the symptoms was available, thus no punch or large biopsies of the cervix have been performed. Seriated ultrasound scans described multiple anechoic cystic lesions within the cervix with a maximum diameter of $35 \mathrm{~mm}$; ancillary a pedunculated myoma sized $55 \mathrm{~mm}$ has been noted. Corpus of the uterus and the ovaries were normal. Color-flow Doppler velocimetry failed to further characterize the cystic lesions, in fact no intralesional vascularity has been noted. No obvious suspect of malignancy has been raised and "multiple retention cysts" was the final report of the scans. In literature there are cases of Nabothian cysts sized up to $40 \mathrm{~mm}$ [12] and a case of obstruction to vaginal labour due to the size of the cyst [13]. Tunnel cluster (TC) is a type of retention cyst, characterised by complex multicystic dilatation of the endocervical glands, occasionally with mucoid discharge. TC is found almost exclusively during pregnancy and can persist for a variable period of time. Although such diagnostic option hasn't been considered before surgery, we precise that the last pregnancy of this patient was more than ten years before. Subsequently an MRI of the pelvis has been performed to confirm the presence of the cysts and to characterize their nature. MRI assessment of the pelvis showed the cystic lesions within the supra-vaginalis portion of the cervix with an intraabdominal growth (Figure 1), hence the multiple negative gynecological and specular examinations.

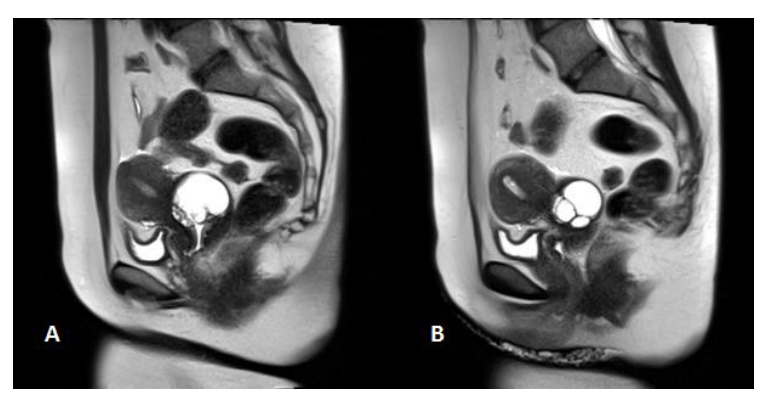

Figure $1 \mathrm{MRI}$ of the pelvis previous surgery. On the left (A) the main cystic lesion; on the right (B) a lateral view with the multiple cystic aspect

Since the persistence of the symptoms and the ancillary presence of the pedunculated myoma (bigger than $5 \mathrm{~cm}$ ) the patient underwent a laparatomy with a total extrafascial hysterectomy and consensual bilateral salpingooophorectomy. Surgery has been accomplished without any intra neither post-operative complications and patient has been discharged after 4 days. Histopathology report of the cervix described an endocervical glandular proliferative lesion with architectural complexity compatible with lobular endocervical gland hyperplasia (LEGH). However, due the invasion of more than half of the cervical stroma a minimal deviation adenocarcinoma cannot be excluded. Immunohistochemical stains displayed glands with cells positive for CEA, p16, estrogen receptor and progestin receptor. Endometrium, corpus of the uterus with the pedunculated myoma, tubes and ovaries have been described as normal. The case has been therefore referred to our Gynecological Oncology service. Review of pathological specimens by multiple pathologists confirmed diagnosis of MDA. Pathological assessment revealed a MUC6 immunohistochemistry indicating a gastric phenotype of the tumor compatible with MDA and with a maximum tumoral size of $23 \mathrm{~mm}$. Multidisciplinary Tumor Board (Radiologist, Gynecology, Radiotherapist, Medical oncology and Pathologist) suggested, according to the available data, a provisional FIGO 1b1 stage treated with simple hysterectomy. Hence two therapeutical pathways have been offered to the patient: adjuvant radiotherapy of the whole pelvis with vaginal brachytherapy versus a robotic bilateral parametrectomy with consensual bilateral pelvic lymphnodal dissection (intensive staging). After a thorough counselling the patient decided to proceed with further surgery as proposed. Surgical procedure has been performed two months after the previous hysterectomy. No intra or post-operative complications have been recorded and the recovery went uneventful and the patient has been discharged after three days. Pathological assessment of the parametria and the pelvic lymph nodes failed to show any further localization of the disease, hence a definitive FIGO Ib1 stage has been noted and no further adjuvant treatment was advised. Since the correlation with PJS an assessment of the colon has been carried out with a pancolonscopy that didn't reveal any abnormality. Patient has 
been sent for oncological strict follow-up and after ten months she is cancer free.

\section{Conclusions}

Persistent gynecological symptoms of unexplained origin should be referred to a tertiary hospital with a Gynecological Oncology service before a symptom-relief treatment is delivered. Moreover, if cervical findings on ultrasound scans and MRI suggest presence of multiple cystic lesions in association with key symptoms, MDA of the cervix has to be considered. Since Gynecological Oncology services are continually developing new diagnosis tests and treatment techniques, whilst improving existing ones, multiple treatment options can be offered even in case of incidental findings in a short frame time.

\section{Consent:}

A signed consent of the patient has been granted for research and accademic purposes, including publication of this case report.

\section{Authors contributions}

$F F, M R, V Z$ and FO were all involved in the management of the disease.

Acknowledgement: None

\section{References}

1. Wells $M$, Ostor $A G$, Crum $C P$, Franceschi $S$, Tommasino $M$, et al. (2003) Tumors of the uterine cervix: Epithelial tumors. Tumors of the breast and female genital organs, World Organization Classification of Tumors. Lyon: IARC Press.

2. Gotoh T, Kikuchi Y, Takano M, Kita T, Oqata S, et al. (2002) An extremely rare case of adenoma malignum with large cystic tumor which resulted in urinary obstruction. Gynecol Oncol 11: 339-343.
3. Gilks CB, Young RH, Aguirre P, DeLellis RA, Scully RE (1989) Adenoma Malignum (minimal deviation adenocarcinoma) of the uterine cervix: A clinicopathological and immunohistochemical analysis of 26 cases. Am J Surg Pathol 13: 717-729.

4. Mills KE, Shuen P, Zolis L (2015) Adenoma Malignum presenting with profound hyponatremia. J Obstet Gynaecol Can 37: 624-627.

5. Mowat A, Land R (2014) Adenoma Malignum presenting as urinary incontinence. Int Urogynecol J 25: 1287-1289.

6. Kaku T, Hachisuga T, Toyoshima S, Enjoji M, Mori T, et al. (1985) Extremely well-differentiated adenocarcinoma ("Adenoma Malignum") of the cervix in a patient with Peutz-Jeghers syndrome. Int J Gynecol Pathol 4: 266-273.

7. Ishii K, Katsuyama T, Ota H, Watanabe T, Matsuyama I, et al. (1999) Cytologic and cytochemical features of adenoma Malignum of the uterine cervix. Cancer 87: 245-53.

8. Tsuda H, Mikami Y, Kaku T, Akiyama F, Hasegawa T, et al. (2003) Inter observer variation in the diagnosis of adenoma Malignum (minimal deviation adenocarcinoma) of the uterine cervix. Pathol Int 53: 440-449.

9. Carleton C, Hoang L, Sah S, Kiyokawa T, Karamurzin YS, et al. (2015) A detailed immunohistochemical analysis of a large series of cervical and vaginal gastric-type adenocarcinomas. Am J Surg Pathol 40: 636-644.

10. Clement PB, Young RH (1989) Deep nabothian cysts of the uterine cervix. A possible source of confusion with minimaldeviation adenocarcinoma (Adenoma Malignum). Int J Gynecol Pathol 8: 340-348.

11. Gagnier J, Kienle G, Altman DG, Moher D, Sox H, et al. (2013) The CARE guidelines: Consensus-based clinical case report guideline development. J Clin Epidemiol 67: 46-51.

12. Vural F, Sanverdi I, Coskun AD, Kusgöz A, Temel O (2015) Large Nabothian cyst obstructing labour passage. J Clin Diagn Res 9: QD06-7.

13. Bin Park S, Lee JH, Lee YH, Song MJ, Choi HJ (2010) Multilocular cystic lesions in the uterine cervix: broad spectrum of imaging features and pathologic correlation. AJR Am J Roentgenol 195: 517-23. 